Article

\title{
Visible Light-Cured Antibacterial Collagen Hydrogel Containing Water-Solubilized Triclosan for Improved Wound Healing
}

\author{
Longhao Jin ${ }^{1,+}$, Kyeongsoon Park ${ }^{2,+} \mathbb{D}$, Yihyun Yoon ${ }^{3}$, Hyeon Soo Kim ${ }^{3}$, Hyeon Ji Kim ${ }^{2}$, Jae Won Choi ${ }^{4}$, \\ Deuk Yong Lee ${ }^{5}$, Heung Jae Chun ${ }^{3,6}$ and Dae Hyeok Yang ${ }^{3, *(D)}$ \\ 1 Department of Orthopedic Surgery, Yanbian University Hospital, Yanji 133000, China; jlh0423@sina.com \\ 2 Department of Systems Biotechnology, Chung-Ang University, Anseong 17546, Gyeonggi, Korea; \\ kspark1223@cau.ac.kr (K.P.); kk07gg@cau.ac.kr (H.J.K.) \\ 3 Institute of Cell and Tissue Engineering, College of Medicine, The Catholic University of Korea, \\ Seoul 06591, Korea; dosk9997@catholic.ac.kr (Y.Y.); 10ksu@catholic.ac.kr (H.S.K.); \\ chunhj@catholic.ac.kr (H.J.C.) \\ 4 Lumenbio Co. Ltd., Seoul 08590, Korea; aa@lumenbio.co.kr \\ 5 Department of Biomedical Engineering, Daelim University, Anyang 13916, Gyeonggi, Korea; \\ dylee@daelim.ac.kr \\ 6 Department of Biomedical \& Health Sciences, College of Medicine, The Catholic University of Korea, \\ Seoul 06591, Korea \\ * Correspondence: yangdh@catholic.ac.kr; Tel.: +82-2-2258-7497 \\ + These authors contributed to this work equally.
}

check for updates

Citation: Jin, L.; Park, K.; Yoon, Y.; Kim, H.S.; Kim, H.J.; Choi, J.W.; Lee, D.Y.; Chun, H.J.; Yang, D.H. Visible Light-Cured Antibacterial Collagen Hydrogel Containing Water-Solubilized Triclosan for Improved Wound Healing. Materials 2021, 14, 2270. https://doi.org/ $10.3390 /$ ma14092270

Academic Editor: Maurice N. Collins

Received: 16 March 2021

Accepted: 25 April 2021

Published: 27 April 2021

Publisher's Note: MDPI stays neutral with regard to jurisdictional claims in published maps and institutional affiliations.

Copyright: (c) 2021 by the authors. Licensee MDPI, Basel, Switzerland.

This article is an open access article distributed under the terms and conditions of the Creative Commons Attribution (CC BY) license (https:// creativecommons.org/licenses/by/ $4.0 /)$.

\begin{abstract}
Infection is one of several factors that can delay normal wound healing. Antibacterial wound dressings can therefore promote normal wound healing. In this study, we prepared an antibacterial wound dressing, consisting of visible light-cured methacrylated collagen (ColMA) hydrogel and a 2-hydroxypropyl-beta-cyclodextrin (HP- $\beta-\mathrm{CD}) /$ triclosan (TCS) complex (CD-icTCS), and evaluated its wound healing effects in vivo. The ${ }^{1} \mathrm{H}$ NMR spectra of ColMA and CDic-TCS revealed characteristic peaks at 1.73, 5.55, 5.94, 6.43, 6.64, 6.84, 6.95, 7.31, and 7.55 ppm, indicating successful preparation of the two material types. In addition, ultraviolet-visible (UV-vis) spectroscopy proved an inclusion complex formation between HP- $\beta$-CD and TCS, judging by a unique peak observed at $280 \mathrm{~cm}^{-1}$. Furthermore, ColMA/CD-ic-TCS exhibited an interconnected porous structure, controlled release of TCS, good biocompatibility, and antibacterial activity. By in vivo animal testing, we found that ColMA/CD-ic-TCS had a superior wound healing capacity, compared to the other hydrocolloids evaluated, due to synergistic interaction between ColMA and CD-ic-TCS. Together, our findings indicate that ColMA/CD-ic-TCS has a clinical potential as an antibacterial wound dressing.
\end{abstract}

Keywords: methacrylated collagen hydrogel; 2-hydroxypropyl-beta-cyclodextrin; triclosan; inclusion complex; antibacterial wound dressing

\section{Introduction}

Wound dressings are widely used to protect the wound bed and accelerate wound healing [1]. For optimal wound healing, the ideal wound dressing should be non-toxic, have suitable viscosity and mechanical properties, and be able to absorb exudate, prevent bacterial infections, and maintain humidity around the wound [2]. 
Hydrogels have many of the properties listed above and can therefore accelerate wound healing [3]. The three-dimensional structure of hydrogels enables them to retain water molecules and thus to provide a moist wound bed environment. This allows for proper cell adhesion and proliferation and thereby accelerates wound healing [4,5]. In addition, the optimal hydration of hydrogels around a wound induces improved autolytic debridement, angiogenesis, and collagen formation [6]. The extent of hydration in hydrogel matrix was characterized by several analytical methods, such as high-resolution magic angle spinning nuclear magnetic resonance (HRMAS-NMR), Fourier-transform infrared attenuated total reflectance (FTIR-ATR) spectroscopy, and Raman spectroscopy [7,8]. Hydrogels, however, also have some disadvantageous mechanical and pharmacokinetic properties. In addition, they lack affinity for cells, which is needed for wound healing $[9,10]$. Collagen is a good wound dressing material as it possesses good biocompatibility, biodegradability, and cytocompatibility [11,12]. However, collagen hydrogels formed by self-assembly are not suitable for soft tissue regeneration due to the low flexibility of the polymer [13]. Various types of collagen hydrogels prepared by physicochemical crosslinking have been introduced, but the relatively high toxicity of the crosslinkers used has limited their biomedical applications [14].

Photopolymerization using visible or ultraviolet light is an attractive method for preparing collagen hydrogels with appropriate mechanical properties, such as photocuring time [15-19]. Therefore, the mechanical strength of the resulting hydrogel, which affects the pharmacokinetic release parameters of drugs/proteins incorporated in the hydrogel, can be controlled [20]. Visible light is safer to use than ultraviolet light. In addition to visible light-cured hydrogel systems, antimicrobial agents as well are accelerators of wound healing. One of them, triclosan, has a broad-spectrum antimicrobial activity and low toxicity to human skin; however, it has poor water solubility [21,22]. In this study, we evaluated the wound healing efficacy of a novel type of antimicrobial dressing agent based on a visible light-cured methacrylated collagen hydrogel containing a betacyclodextrin/triclosan inclusion complex (ColMA/CD-ic-TCS) using a mouse skin defect model (Figure 1). Methacrylated collagen was characterized by proton nuclear magnetic resonance $\left({ }^{1} \mathrm{H}\right.$ NMR). Beta-cyclodextrin $(\beta-\mathrm{CD})$ was used to improve the water-solubility of triclosan (TCS) because of its ability to promote inclusion complex formation. The inclusion complex between $\beta-C D$ and triclosan was analyzed by ${ }^{1} \mathrm{H}$ NMR and UV spectroscopy. Additionally, we performed in vitro biocompatibility testing and in vivo experiments. 


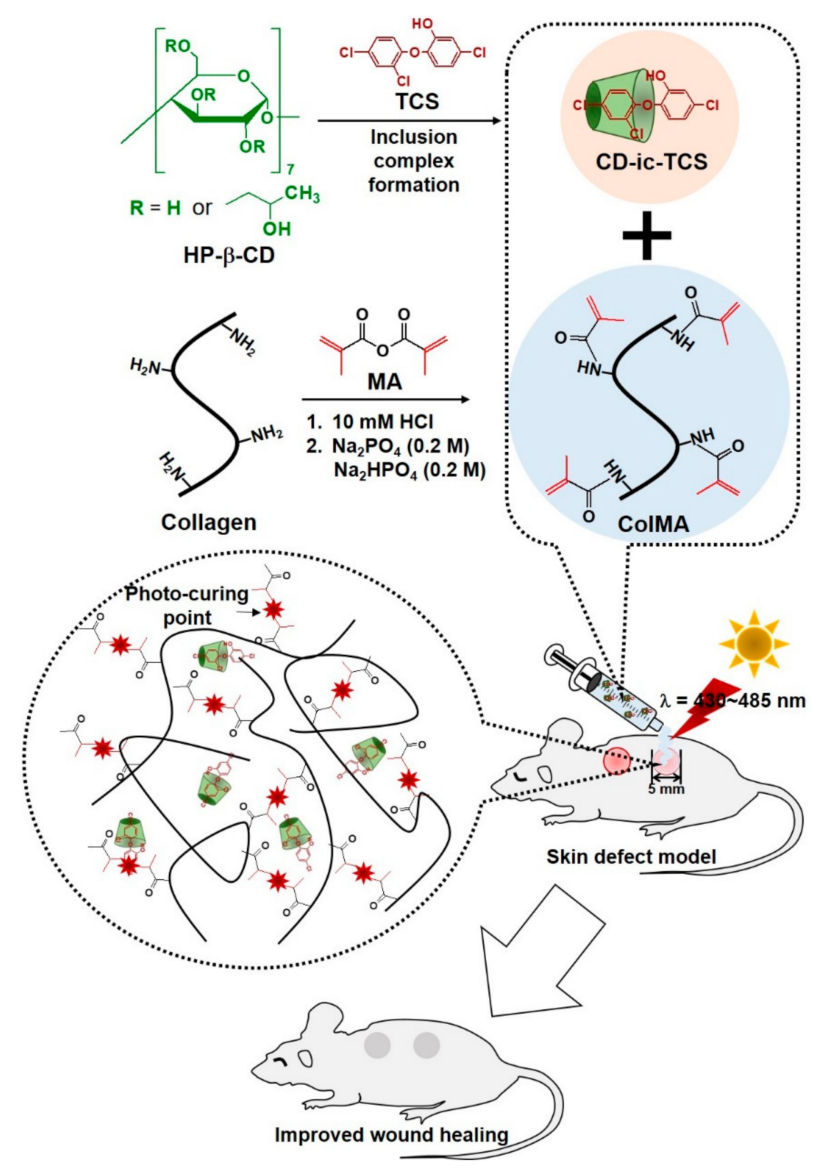

Figure 1. Schematic illustration of the preparation of CD-ic-TCS and ColMA, and the in vivo animal test of ColMA/CD-ic-TCS using a skin defect model. CD-ic-TCS-inclusion complex between 2 hydroxypropyl-beta cyclodextrin (HP- $\beta-\mathrm{CD})$ and triclosan (TCS); ColMA-methacrylated collagen formed by conjugation of methacrylic anhydride (MA) to a collagen backbone; ColMA/CD-ic-TCSCD-ic-TCS-incorporated ColMA hydrogel prepared by visible light irradiation $(\lambda=430-485 \mathrm{~nm})$.

\section{Materials and Methods}

\subsection{Materials}

Collagen was purchased from MSBIO, Inc. (Seongnam, Kyunggi, Korea). Methacrylic anhydride (MA), sodium phosphate monobasic, sodium phosphate dibasic, 2-hydroxypropylbeta-cyclodextrin (HP- $\beta-\mathrm{CD})$, and triclosan (TCS) were obtained from Sigma-Aldrich (St. Louis, MO, USA). Riboflavin 5'-monophosphate sodium salt (riboflavin) as a photoinitiator was obtained from Santa Cruz (Santa Cruz, CA, USA). Dialysis membrane tubes (cut-off: $25 \mathrm{kDa}$ and 3.5-5.0 kDa; Spectrum Laboratories Inc., Rancho Dominguez, CA, USA) were used for methacrylated collagen (ColMA). The NIH3T3 mouse fibroblast cell line was obtained from the Korean Cell Line Bank (Seoul, Korea).

\subsection{Preparation of Methacrylated Collagen (ColMA) Derivative}

ColMA was prepared in three steps as follows [23]. First, collagen (1 g) was dissolved in $10 \mathrm{mM} \mathrm{HCl}$ solution $(300 \mathrm{~mL})$. Then, methacrylic anhydride (MA, $0.5 \mathrm{~g}$ ) was added to the acidic collagen solution, and the reaction then proceeded at $4{ }^{\circ} \mathrm{C}$ for $16 \mathrm{~h}$. The reactant was dialyzed in a $10 \mathrm{mM} \mathrm{HCl}$ solution using a dialysis membrane tube (cutoff: $25 \mathrm{kDa}$ ) for 2 days. Before use, the purified ColMA solution was lyophilized at $-90{ }^{\circ} \mathrm{C}$ using a lyophilizer (FD8508; IlShinBioBase Co. Ltd., Yangju, Kyunggi, Korea). Third, the acidic ColMA solution was neutralized using two solutions, namely $0.2 \mathrm{M}$ sodium phosphate monobasic (solution $\mathrm{A}, \mathrm{NaH}_{2} \mathrm{PO}_{4}$ ) and $0.2 \mathrm{M}$ sodium phosphate dibasic (solution $\mathrm{B}, \mathrm{Na}_{2} \mathrm{HPO}_{4}$ ), for dissolution. Solutions A and B were mixed at a 19:81 ratio 
$(v / v \%)$ (solution C) prior to addition of collagen, and all solutions were stored overnight in a refrigerator $\left(4^{\circ} \mathrm{C}\right)$ before use. Afterward, the three solutions were then mixed at 2 to 1 $(v / v \%)$ or 3 to $1(v / v \%)$ ratios to adjust the $\mathrm{pH}$ to $6.4 \sim 6.8$. Finally, the $\mathrm{pH}$ was adjusted to 7.4 using a $1 \mathrm{~N} \mathrm{NaOH}$ solution $(25-65 \mu \mathrm{L} / \mathrm{mL})$. The final product was analyzed by proton nuclear magnetic resonance ( ${ }^{1} \mathrm{H}$ NMR; Bruker Avance; Bruker Avance 400; Harwell, UK) in the co-solvents of $\mathrm{D}_{2} \mathrm{O}$ and acetic acid- $d 4$.

\subsection{Inclusion Complex between 2-Hydroxypropyl-Beta-Cyclodextrin and Triclosan (CD-ic-TCS)}

HP- $\beta$-CD $(1 \mathrm{~g})$ and TCS $(100 \mathrm{mg})$ were dissolved in distilled water $(10 \mathrm{~mL})$ and acetone $(1 \mathrm{~mL})$, respectively [24]. The TCS solution was slowly dropped in the aqueous HP- $\beta$ $\mathrm{CD}$ solution with continuous agitation, and then the mixture was continuously stirred at room temperature for $24 \mathrm{~h}$ to evaporate the acetone. The complex solution was stored in a refrigerator $\left(2-4{ }^{\circ} \mathrm{C}\right)$ before use. The inclusion complex was analyzed by ${ }^{1} \mathrm{H}$ NMR $\left(\mathrm{D}_{2} \mathrm{O}\right)$ and ultraviolet-visible (UV-vis) spectroscopy (Multiskan ${ }^{\circledR}$ Spectrum; Thermo Fisher Scientific; Waltham, MA, USA) using quartz cuvettes (frosted wall, $0.7 \mathrm{~mL}$ ).

\subsection{Preparation of CD-ic-TCS-Loaded ColMA Hydrogel (ColMA/CD-ic-TCS)}

The complex solution was added to the ColMA hydrogel precursor solution [25]. The two solutions were mixed until a homogeneous mixture was obtained and photo-cured using riboflavin ( $12 \mu \mathrm{M}$; DPBS) as a photoinitiator for $40 \mathrm{~s}$, and then stored overnight in a refrigerator $\left(2 \sim 4{ }^{\circ} \mathrm{C}\right)$ for stabilization. The mixture was transferred into a $1 \mathrm{~mL}$ syringe and stored in a refrigerator $\left(2 \sim 4{ }^{\circ} \mathrm{C}\right)$ before use. SEM (S-3000H, Hitachi, Japan) was employed for observing the morphologies of hydrogel samples. Prior to observation, the samples were attached on metal mounts with a carbon tape and coated with $\mathrm{Au} / \mathrm{Pd}$.

\subsection{In Vitro Release Test}

The release behavior of TCS from the ColMA/TCS and ColMA/CD-ic-TCS hydrogels was analyzed by ultraviolet-visible spectroscopy (Hitachi U-4100, Mountain View, CA, USA) [25]. The amount of TCS in both hydrogels was $10 \mathrm{mg}$. The hydrogels were added to dialysis membrane tubes (cut-off: $3.5-5.0 \mathrm{kDa}$ ) and immersed in PBS (20 mL; pH 7.4) at $37^{\circ} \mathrm{C}$. At each time interval $(1,3,6,12,24,48,72,96,120,144$, and $168 \mathrm{~h}), 2 \mathrm{~mL}$ of PBS was extracted from each sample, and the same volume of fresh PBS was added. This test was performed in triplicate. The absorbance of the extracted PBS was measured at $282 \mathrm{~nm}$.

\subsection{In Vitro Biocompatibility}

In vitro cytotoxicity testing to investigate the biocompatibility of ColMA/CD-ic-TCS was performed using NIH3T3 cells (Korean Cell Line Bank, Seoul, Korea) between passages 10 and 15. As a control, cells were seeded on a 24-well plate and cultured in Dulbecco's Modified Eagle Medium containing $25 \mathrm{mM}$ HEPES, $25 \mathrm{mM} \mathrm{NaHCO}_{3}$, and heat inactivated bovine calf serum. Three kinds of hydrogels, DuoDERM ${ }^{\circledR}$, ColMA (TCS: $1.8 \mu \mathrm{g}$ ), and ColMA/TCS (TCS: $1.8 \mu \mathrm{g}$ ), were placed in a 96-well plate. After the seeding of $1 \times 10^{4}$ cells/well on each hydrogel, the cells were incubated for 1,3, and 7 days in an incubator at $37^{\circ} \mathrm{C}$ and $5 \% \mathrm{CO}_{2}$. At specific time intervals, the cell-seeded hydrogels were treated with CCK-8 $(100 \mu \mathrm{L})$, and this was followed by a $2 \mathrm{~h}$ incubation. After extracting supernatants from the CCK-8-treated wells, the optical densities of the supernatant samples were measured at $450 \mathrm{~nm}$ using a microplate reader (SpectraMax ${ }^{\circledR}$ i3; Molecular Devices, Sunnyvale, CA, USA) [25].

\subsection{Antibacterial Assay}

Antibacterial activity was evaluated according to a previous study [25,26]. Five hundred microliters of diluted S. aureus and E. coli $\left(1 \times 10^{6}\right.$ bacterial $\left./ \mathrm{mL}\right)$ were added to the wells of a 48-well plate containing DuoDERM ${ }^{\circledR}$, ColMA, ColMA/TCS, or ColMA/CDic-TCS hydrogels (TCS: $1.8 \mu \mathrm{g}$ ), and the plates were incubated at $37^{\circ} \mathrm{C}$ for $2 \mathrm{~h}$. Then, $1 \mathrm{~mL}$ of PBS was added to each well to resuspend live bacteria. Aliquots of $100 \mu \mathrm{L}$ of the 
suspension from each well were plated on Luria-Bertani agar (LB agar) plates, and these plates were incubated at $37^{\circ} \mathrm{C}$ for $24 \mathrm{~h}$. Numbers of bacterial colonies were recorded, and kill percentage was calculated as follows:

$$
\text { Kill } \%=\{(\text { cell count of control-survivor of hydrogels }) / \text { cell of control }\} \times 100
$$

\subsection{In Vivo Animal Testing}

Animal experiments were approved by the Institutional Animal Care and Use Committee of Chung-Ang University (13 April 2020; 2020-00037) [21]. Twenty Balb/C mice (DooYeol Biotech.; Seoul, Korea) of average body weight $(20 \mathrm{~g}, n=4)$ were used to evaluate wound healing efficacy. The mice were divided into five groups: control, commercially available DuoDERM ${ }^{\circledR}$ gel, ColMA, ColMA/TCS, and ColMA/HPCD-ic-TCS. Hair on the back of the mice was removed with an electrical shaver and hair removal cream. Four circular defects $(5 \mathrm{~mm})$ on the back of each mouse were produced using biopsy punches. Prior to the sample treatment of the defects, the hydrogel precursor solutions were transferred to $1 \mathrm{~mL}$ syringes. The syringes were used to place $100 \mu \mathrm{L}$ of a hydrogel solution on the defects. This step was followed by photo-curing with blue light irradiation for $10 \mathrm{~s}$. At predetermined time intervals $(1,7$, and 10 days), hydrogel-treated defects were extracted, and gross appearance was observed. The size of the remaining wound was measured using Image J software (National Institutes of Health Bethesda, MD, USA). Extracted skin was fixed in $10 \%$ formalin solution and evaluated by hematoxylin and eosin (H\&E) and Masson's trichrome staining.

\subsection{Histological Evaluation}

The formalin-fixed skin tissue containing wounds obtained at 1, 7, and 14 days after wound creation was dehydrated using a series of ethanol solutions [21]. The dehydrated tissues were embedded in paraffin and sectioned to a thickness of $3 \mu \mathrm{m}$ using a microtome (DSC1; Leica, Wetzlar, Germany). Afterward, sectioned slides were stained using H\&E and Masson's trichrome stains. These sections were observed using a slide scanner (Pannoramic MIDI; 3DHISTECH Ltd., Budapest, Hungary) at $2.0 \times$ and $7.0 \times$ and a panoramic viewer (Version 1.15.3; Pannoramic MIDI; 3DHISTECH Ltd., Budapest, Hungary) program.

\subsection{Statistical Analysis}

In vitro biocompatibility, antibacterial activity, TCS release, and wound size were evaluated three times, and results were expressed as the mean \pm standard deviation of these triplicate measurements. One-way analysis of variance (ANOVA), as implemented in SPSS (SPSS Inc., Chicago, IL, USA), was used to assess the statistical significance of differences among groups $\left({ }^{*} p<0.05\right)$.

\section{Results}

\section{1. ${ }^{1} H$ NMR Spectra of CD-ic-TCS and ColMA}

The inclusion complex between HP- $\beta-C D$ and TCS and the chemical reaction between collagen and MA were characterized by ${ }^{1} \mathrm{H}$ NMR analysis using $\mathrm{D}_{2} \mathrm{O}$ (Figure 2). As shown in Figure $2 \mathrm{~A}$, the ${ }^{1} \mathrm{H}$ NMR spectrum of CD-ic-TCS was compared to those of HP- $\beta$-CD and TCS. HP- $\beta-C D$ exhibited an H-1 peak at 4.91 ppm and H-2,3,4,5,6 peaks at 3.12$3.98 \mathrm{ppm}$ [27]. In the ${ }^{1} \mathrm{H}$ NMR of TCS, six peaks at $6.43,6.64,6.84,6.95,7.31$, and $7.55 \mathrm{ppm}$ were observed [27], corresponding to the ring structure of TCS. CD-ic-TCS had ${ }^{1} \mathrm{H}$ NMR characteristics of both HP- $\beta-C D$ and TCS. We attributed the appearance of the TCS-related peaks in the ${ }^{1} \mathrm{H}$ NMR spectrum of CD-ic-TCS to water solubilization of TCS by the inclusion complex formation between HP- $\beta$-CD and TCS. The ${ }^{1} \mathrm{H}$ NMR spectrum of ColMA is shown in Figure 2B. Compared to the ${ }^{1} \mathrm{H}$ NMR spectrum of collagen, new peaks at 1.73, 5.55, and $5.94 \mathrm{ppm}$ were observed for ColMA, which we attributed to the methacrylation containing methyl and vinyl groups. The integration ratio of 5.88 to $5.12 \mathrm{ppm}$ indicated that the substitution degree of methylation was $70 \%$. 


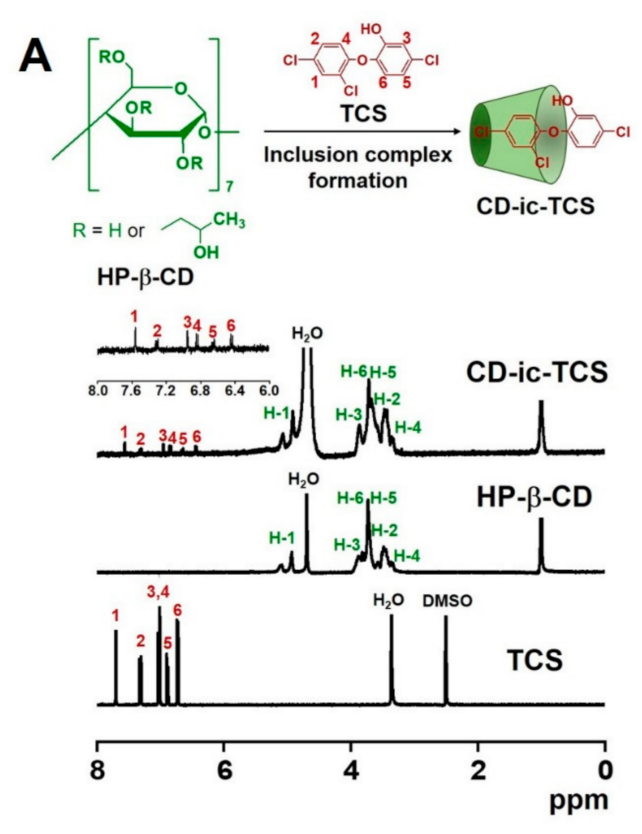

B
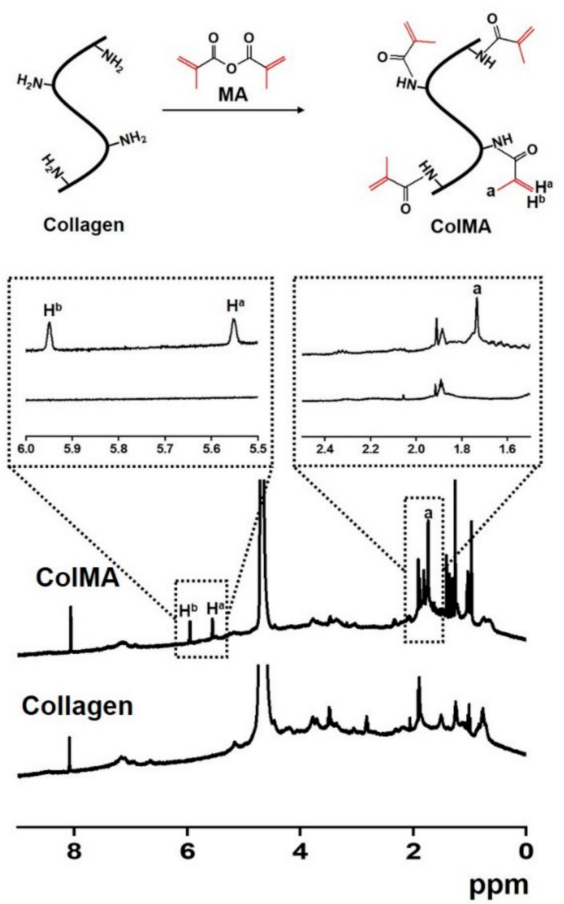

Figure 2. ${ }^{1} \mathrm{H}$ NMR spectra of (A) TCS, HP- $\beta-C D$, and CD-ic-TCS; and (B) collagen and ColMA. TCS was analyzed using DMSO- ${ }_{d 6}$. The ${ }^{1} \mathrm{H}$ NMR analyses of HP- $\beta-C D$ and CD-ic-TCS were performed using $\mathrm{D}_{2} \mathrm{O}$. Collagen and ColMA were analyzed using the co-solvents of $\mathrm{D}_{2} \mathrm{O}$ and acetic acid- $d 4$.

\subsection{UV Spectrum of CD-ic-TCS}

The inclusion complex formation between HP- $\beta-C D$ and TCS was further investigated by UV-vis spectroscopy performed in the wavelength range from 200 to $400 \mathrm{~cm}^{-1}$ (Figure 3). In aqueous solution, no absorption peak of TCS was detected due to its poor water solubility. By contrast, TCS dissolved in EtOH exhibited an absorption peak at $282 \mathrm{~cm}^{-1}$ [28]. No absorption peak of HP- $\beta-C D$ was found in the monitored wavelength range. CD-ic-TCS showed an absorption peak of TCS at $280 \mathrm{~cm}^{-1}$ in an aqueous solution. These results indicated that CD-ic-TCS contributed to the water solubilization of TCS by the inclusion complex formation with HP- $\beta$-CD. 


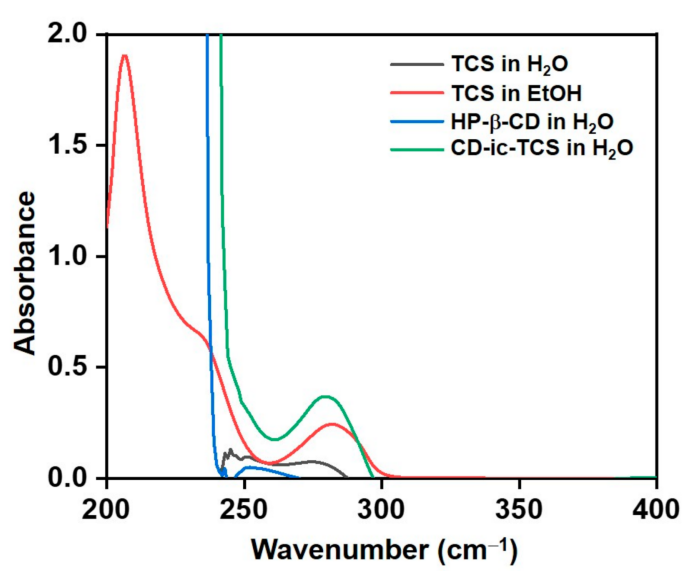

Figure 3. UV spectra of TCS in $\mathrm{H}_{2} \mathrm{O}$, TCS in EtOH, HP- $\beta-C D$ in $\mathrm{H}_{2} \mathrm{O}$, and CD-ic-TCS in $\mathrm{H}_{2} \mathrm{O}$. The spectra were monitored from 200 to $400 \mathrm{~cm}^{-1}$. TCS-triclosan; HP- $\beta$-CD-2-hydroxypropyl-betacyclodextrin; CD-ic-TCS-inclusion complex between HP- $\beta$-CD and TCS.

\subsection{SEM Images, TCS Release, and Cell Proliferation}

The microstructural morphologies of ColMA, ColMA/TCS, and ColMA/CD-ic-TCS were investigated by scanning electron microscopy (SEM). Figure 4 shows SEM images of freeze-dried ColMA, ColMA/TCS, and ColMA/CD-ic-TCS observed at $\times 500$. The samples had 3-D networks with interconnected macropores. This may be attributed to the 3-D hydrogel network formation by visible light photo-curing. The interconnected porous structure of hydrogels plays a significant role in their drug release behavior. Figure 5 shows the release behavior of TCS in ColMA/TCS and ColMA/CD-ic-TCS incubated at $37^{\circ} \mathrm{C}$ for predetermined time intervals $(1,3,6,12,24,48,72,96,120,144$, and $168 \mathrm{~h})$. The hydrogels showed two release patterns: an initial burst followed by a controlled release. ColMA/TCS exhibited an initial burst release for $48 \mathrm{~h}$ with a controlled release sustained thereafter. Although ColMA/CD-ic-TCS showed a controlled release in a sustained manner along with an initial $24 \mathrm{~h}$ burst, it had faster release behavior than ColMA/TCS due to the improved water solubility of TCS by the inclusion complex formation with HP- $\beta$-CD. In addition to the drug release behavior, an interconnected porosity provides a more favorable platform for cell proliferation. The proliferation of NIH3T3 cells treated with TCS, ColMA/TCS, or ColMA/CD-ic-TCS for 1,3, and 7 days is shown in Figure 6. The cell proliferation of all samples increased gradually as a function of culture time. In addition, the ColMA-based samples exhibited a remarkable cell proliferation as compared to DuoDERM ${ }^{\circledR}$ over time. Among ColMA-based samples, ColMA/CD-ic-TCS treatment was associated with the highest cell proliferation. These results indicated that ColMA/CD-ic-TCS had a superior biocompatibility.
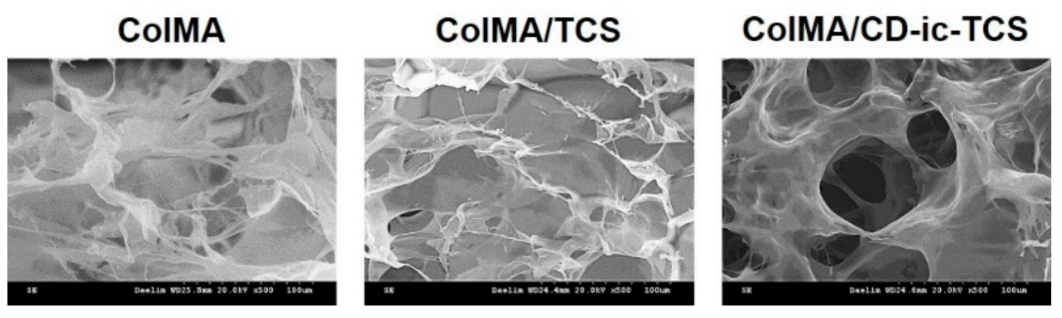

Figure 4. Morphologies of ColMA, ColMA/TCS, or ColMA/CD-ic-TCS observed by SEM at $\times 500$. ColMA - methacrylated collagen; ColMA/TCS—-triclosan (TCS) incorporated ColMA; ColMA/CDic-TCS-HP- $\beta$-CD/TCS complex incorporated ColMA. 


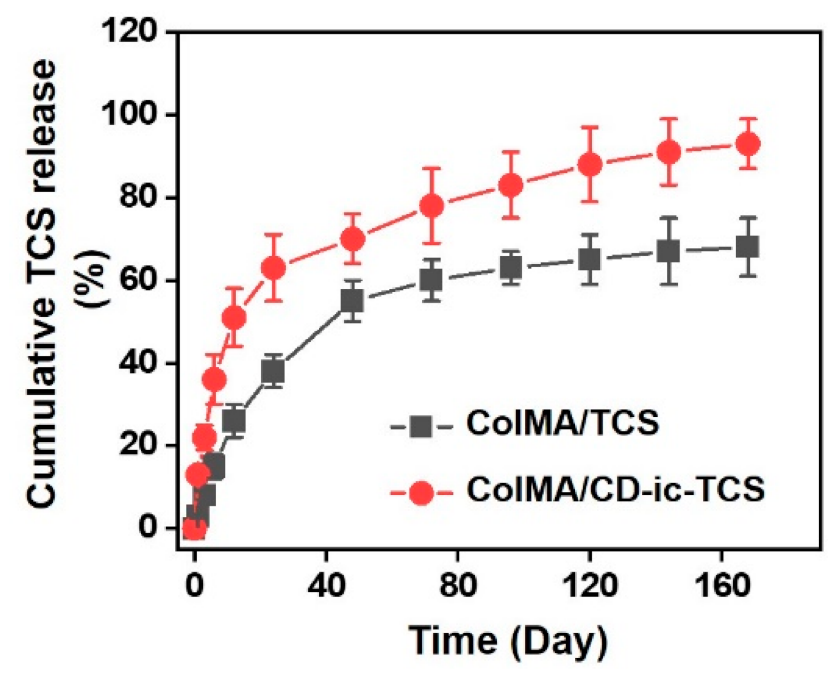

Figure 5. In vitro release behavior of TCS in ColMA/TCS and ColMA/CD-ic-TCS. This test was carried out in triplicate at $37^{\circ} \mathrm{C}$, and the cumulative TCS release percentage was determined at each of the chosen time intervals $(1,3,6,12,24,48,72,96,120,144$, and $168 \mathrm{~h})$. ColMA/TCS-triclosan (TCS) incorporated ColMA; ColMA/CD-ic-TCS-HP- $\beta$-CD/TCS complex incorporated ColMA. This experiment was performed in triplicate $(n=3)$. Results were expressed as mean \pm standard deviation.

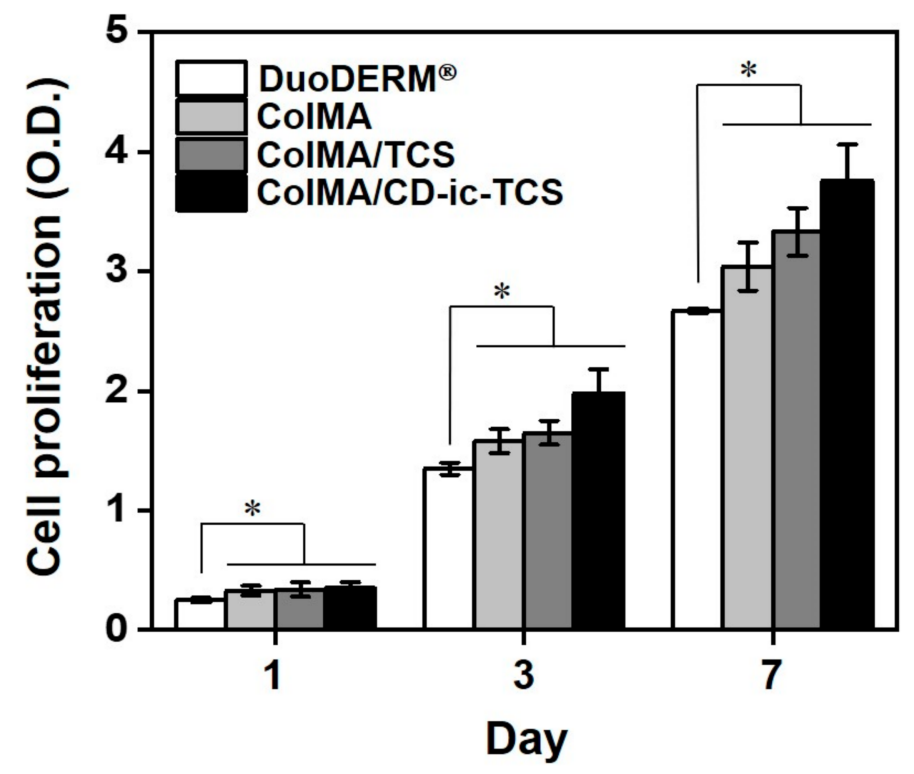

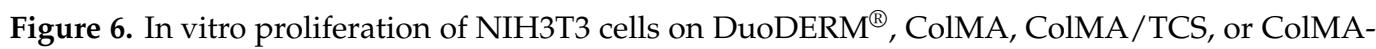
CD-ic-TCS for culture periods of 1,3, and 7 days. ColMA-methacrylated collagen; ColMA/TCStriclosan (TCS) incorporated ColMA; ColMA/CD-ic-TCS-HP- $\beta$-CD/TCS complex incorporated ColMA. This experiment was performed in triplicate $(n=3)$. Results were expressed as mean \pm standard deviation $\left({ }^{*} p<0.05\right)$.

\subsection{Antibacterial Activity}

The antibacterial activity of ColMA/CD-ic-TCS against E. coli and S. aureus over $48 \mathrm{~h}$ was compared to that of DuoDERM ${ }^{\circledR}$, TCS, ColMA, and ColMA/TCS (Figure 7). The samples treated with DuoDERM ${ }^{\circledR}$ or ColMA showed high kill percentages of the bacteria after the $48 \mathrm{~h}$. On the contrary, TCS-related samples had lower kill percentages than DuoDERM $^{\circledR}$ and ColMA. The kill percentage of ColMA/TCS-treated samples remained around $77-78 \%$ after $48 \mathrm{~h}$. Moreover, ColMA/CD-ic-TCS-treated samples had the highest kill percentage among all samples, indicated that TCS would increase the kill percentages of E. coli and S. aureus. 

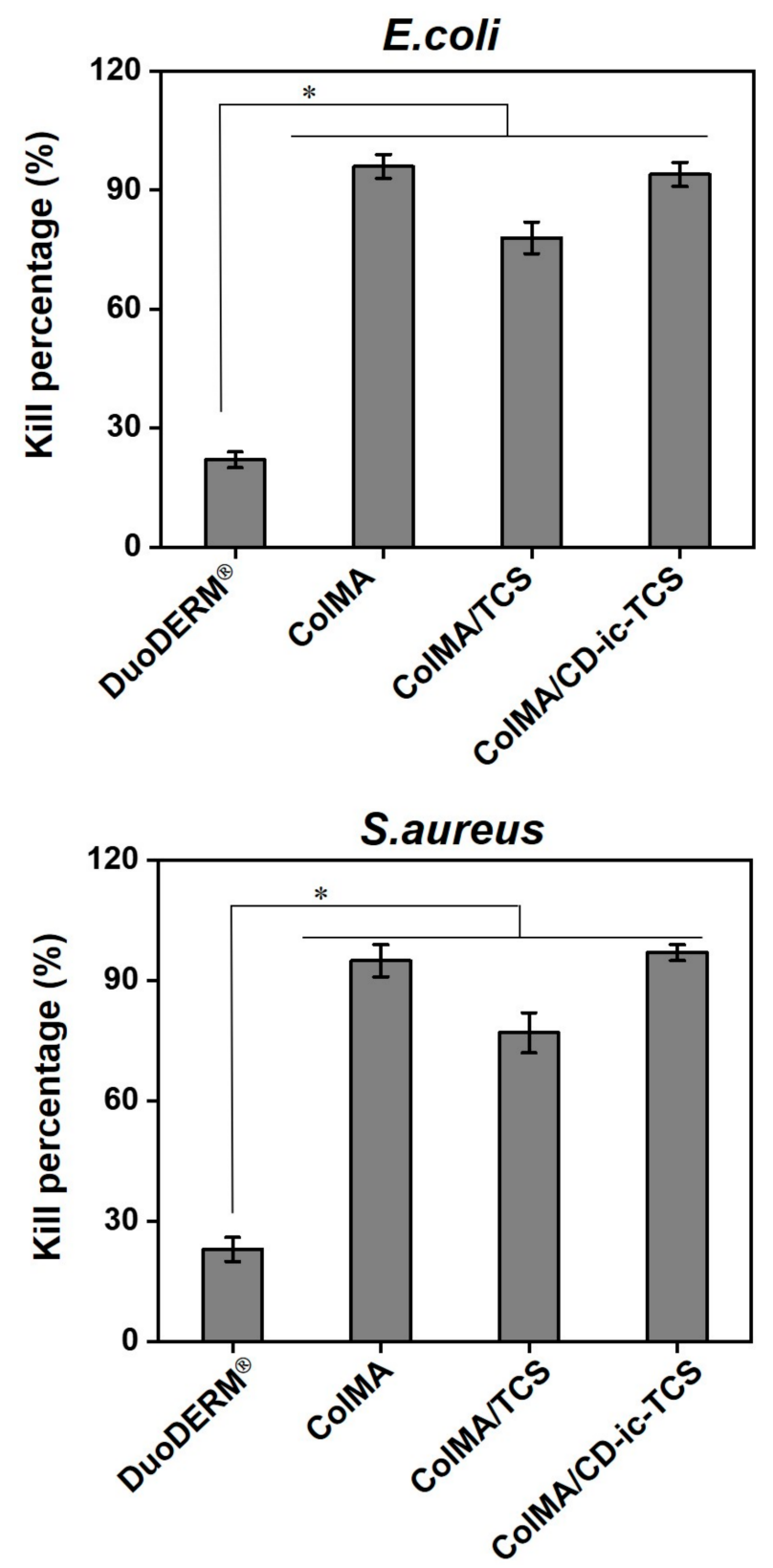

Figure 7. Antibacterial activity of DuoDERM ${ }^{\circledR}$, ColMA, ColMA/TCS, and ColMA-CD-ic-TCS against E.coli and S. aureus. ColMA-methacrylated collagen; ColMA/TCS—triclosan (TCS) incorporated ColMA; ColMA/CD-ic-TCS-HP- $\beta-C D / T C S$ complex incorporated ColMA. This experiment was performed in triplicate $(n=3)$. Results were expressed as mean \pm standard deviation $\left({ }^{*} p<0.05\right)$.

\subsection{Gross Appearances and Wound Healing}

The gross appearance and remaining size of the control and the DuoDERM ${ }^{\circledR}{ }_{-}$, ColMA-, ColMA/TCS-, and ColMA/CD-ic-TCS-treated wounds were investigated after punching, on days 1, 4, 7, 10, and 14 (Figure 8). As shown in Figure 8A, the wound size decreased in all groups over time. Furthermore, compared with the control, DuoDERM ${ }^{\circledR}$, and ColMA samples, full skin-covered wound healing was observed in the ColMA and ColMA/CD-icTCS groups. Further examination of wound healing as a function of time was performed (Figure 8B,C). On day 1, mice in the ColMA/CD-ic-TCS group showed more acceler-

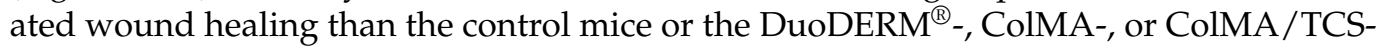
treated mice. On day 3, accelerated wound healing was observed in the hydrogel-treated wounds. In addition, the ColMA/CD-ic-TCS mice showed better wound healing than 
the DuoDERM ${ }^{\circledR}$, ColMA, and ColMA/TCS mice. Like the results obtained on day 7, the wound healing of the hydrogel-treated mice on day 10 was better than that of the control mice. ColMA/CD-ic-TCS had the greatest wound healing effect among all treatments over 7 days. After day 7, all samples showed similar wound healing. We confirmed that ColMA and the water solubility of TCS accelerated wound healing.
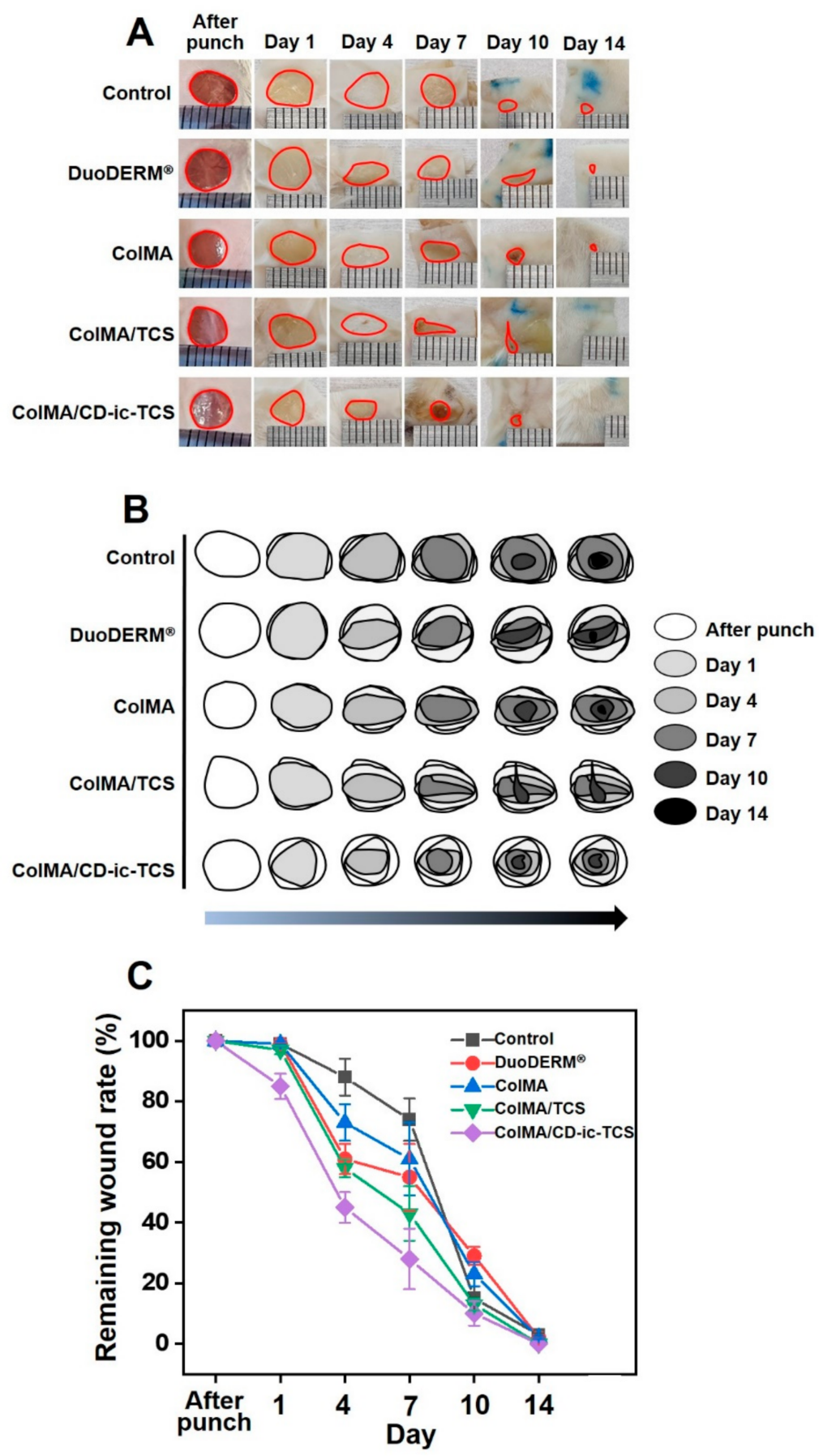

Figure 8. (A) Gross appearances of wound defects of control wounds and those treated with DuoDERM $^{\circledR}$, ColMA, ColMA/TCS, or ColMA/CD-ic-TCS for 1, 4, 7, 10, and 14 days. (B) Schematic diagram of remaining wound sizes at each time interval. (C) Remaining wound rates calculated at each time interval. This experiment was performed three times $(n=3)$. Results were expressed as mean \pm standard deviation. ColMA - methacrylated collagen; ColMA/TCS-triclosan (TCS) incorporated ColMA; ColMA/CD-ic-TCS-HP- $\beta$-CD/TCS complex incorporated ColMA. 


\subsection{Histological Evaluations}

Figure 9 shows H\&E-stained slides of wound defects treated with DuoDERM ${ }^{\circledR}$, ColMA, ColMA/TCS, or ColMA/CD-ic-TCS, compared to an untreated wound (control). On day 1 , inflammatory cells and a provisional extracellular matrix were observed in all groups. Epidermis production began in the wounds of hydrogel-treated groups (DuoDERM $^{\circledR}$, ColMA, ColMA/TCS, and ColMA/CD-ic-TCS). The ColMA/CD-ic-TCS mice showed remarkable epidermis production and angiogenesis, indicating an acceleration of wound healing. On day 7, the inflammatory cells were still observed in all groups. However, all groups also had a fully covered epidermis and a granulation tissue. At this time, compared with the hydrogel-treated groups, the control group exhibited scanty epidermal regeneration in the center of the wound and new blood vessels formation at the wound edges. Similarly to the control, the DuoDERM ${ }^{\circledR}$-treated mice also had wounds fully covered by epidermis and granulation tissue, and angiogenesis around the wound edges was observed. However, the granulation tissue was thicker in the DuoDERM ${ }^{\circledR}$ group than in the control group. The mice treated with ColMA-based hydrogels showed greater wound healing acceleration than those treated with DuoDERM ${ }^{\circledR}$. The ColMA-based hydrogels induced a thinner epithelial layer than DuoDERM ${ }^{\circledR}$. In the case of the hydrogels, ColMA induced angiogenesis around the edges of the wound, whereas angiogenesis was generally observed in the granulation tissues in the ColMA/TCS- and ColMA/CD-ic-TCStreated groups. Furthermore, the wounds treated with TCS-loaded hydrogels produced hair follicles. On day 14, the wounds treated with ColMA-based hydrogels showed an accelerated wound healing compared to the control and DuoDERM ${ }^{\circledR}$-treated wounds. The hydrogels contributed to the production of hair follicles and sebaceous glands. Moreover, ColMA/CD-ic-TCS promoted wound recovery.

Day 1
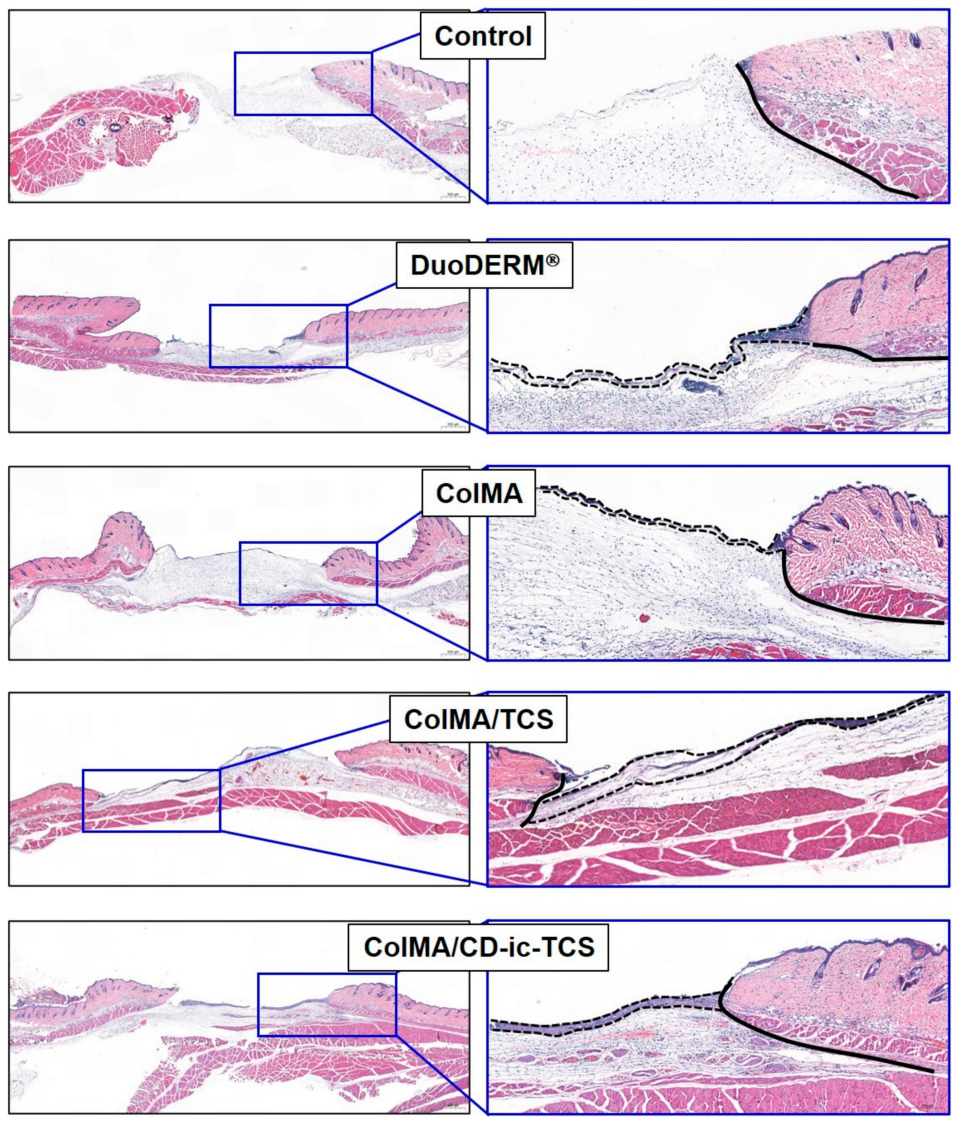

Figure 9. Cont. 


\section{Day 7}
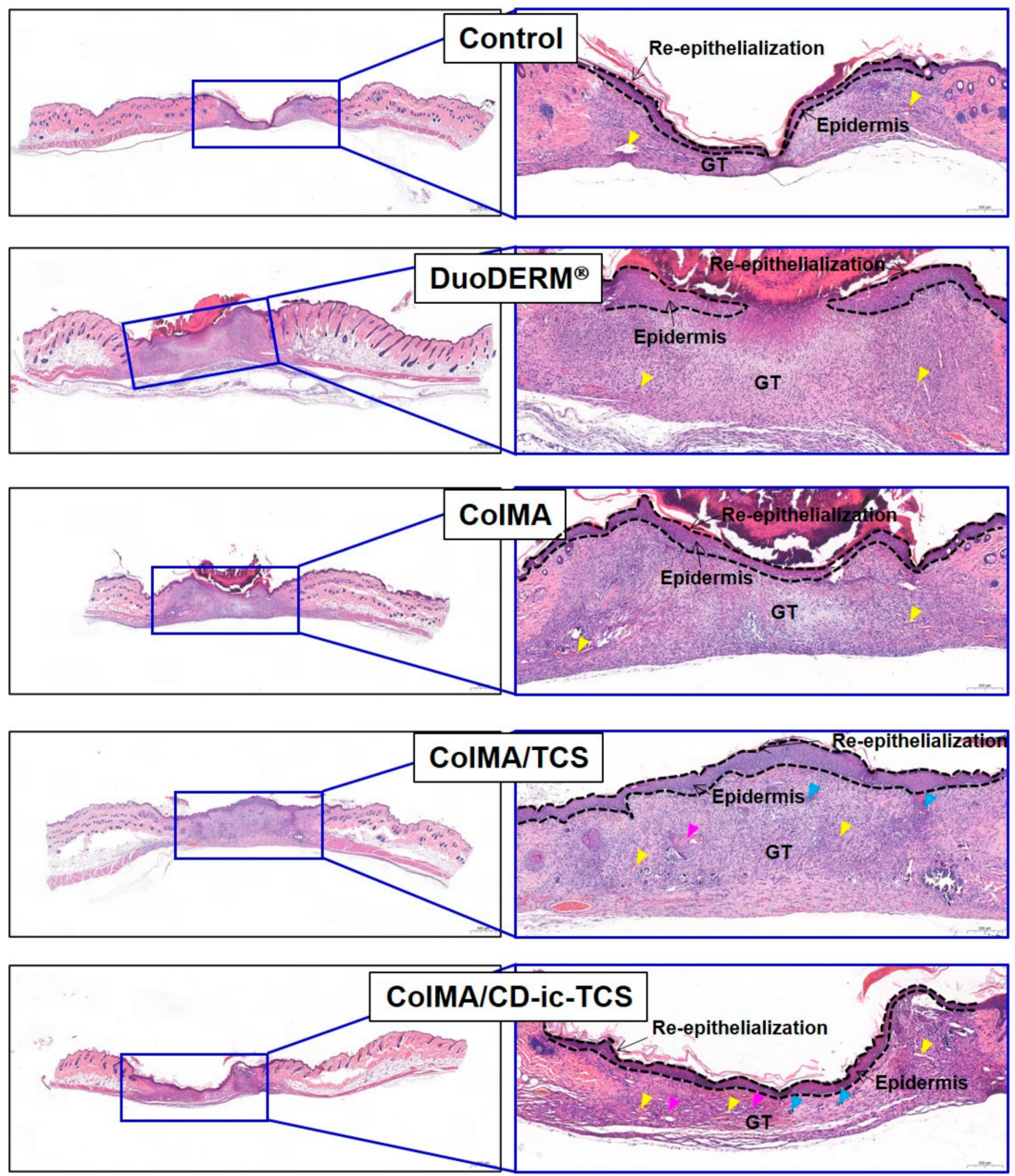

Figure 9. Cont. 


\section{Day 14}
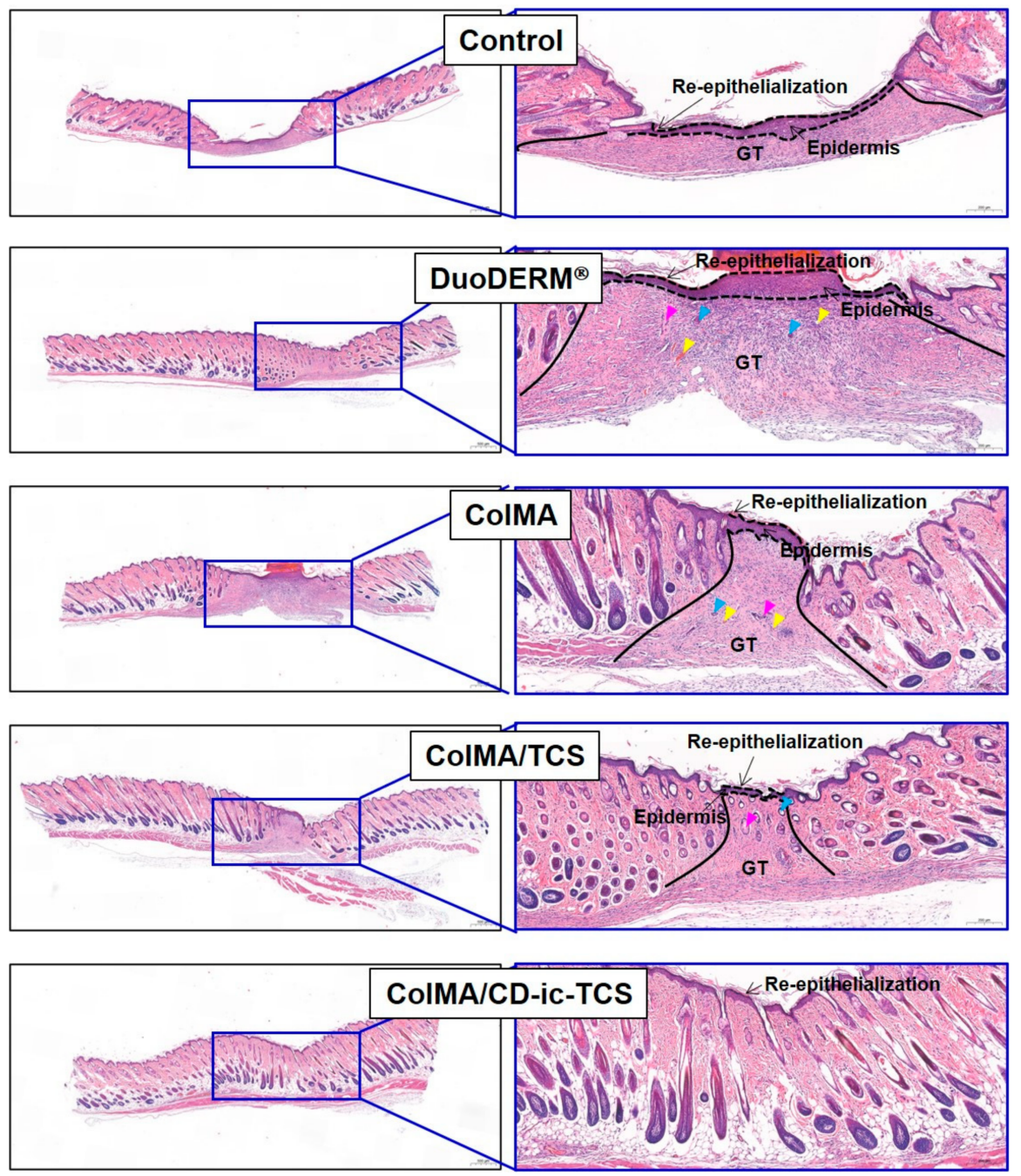

Figure 9. H\&E-stained slides of wound defects treated with DuoDERM ${ }^{\circledR}$, ColMA, ColMA/TCS, or ColMA/CD-ic-TCS for 1, 7, and 14 days. The (left) and (right) images were observed at $2.0 \times(500 \mu \mathrm{m})$ and $7.0 \times(200 \mu \mathrm{m})$, respectively. ColMA/TCS—-triclosan (TCS) incorporated ColMA; ColMA/CD-icTCS-HP- $\beta$-CD/TCS complex incorporated ColMA; GT-granulation tissue. Spaces in black and dotted lines indicate the epidermis and GT, respectively.

Collagen synthesis in the control and hydrogel-treated wounds was investigated by Masson's trichrome staining (Figure 10). On days 1 and 7, no differences in collagen synthesis were observed among samples. On day 14, collagen synthesis was observed in all samples. Compared with the control and DuoDERM ${ }^{\circledR}$-treated samples, those treated with the ColMA-based hydrogels showed an advanced wound healing. In addition, incorporation of TCS in ColMA further enhanced wound healing. We attributed some of the acceleration of wound healing to the improvement of the water solubility of TCS by inclusion complex formation with HP- $\beta-\mathrm{CD}$. 


\section{Day 1}
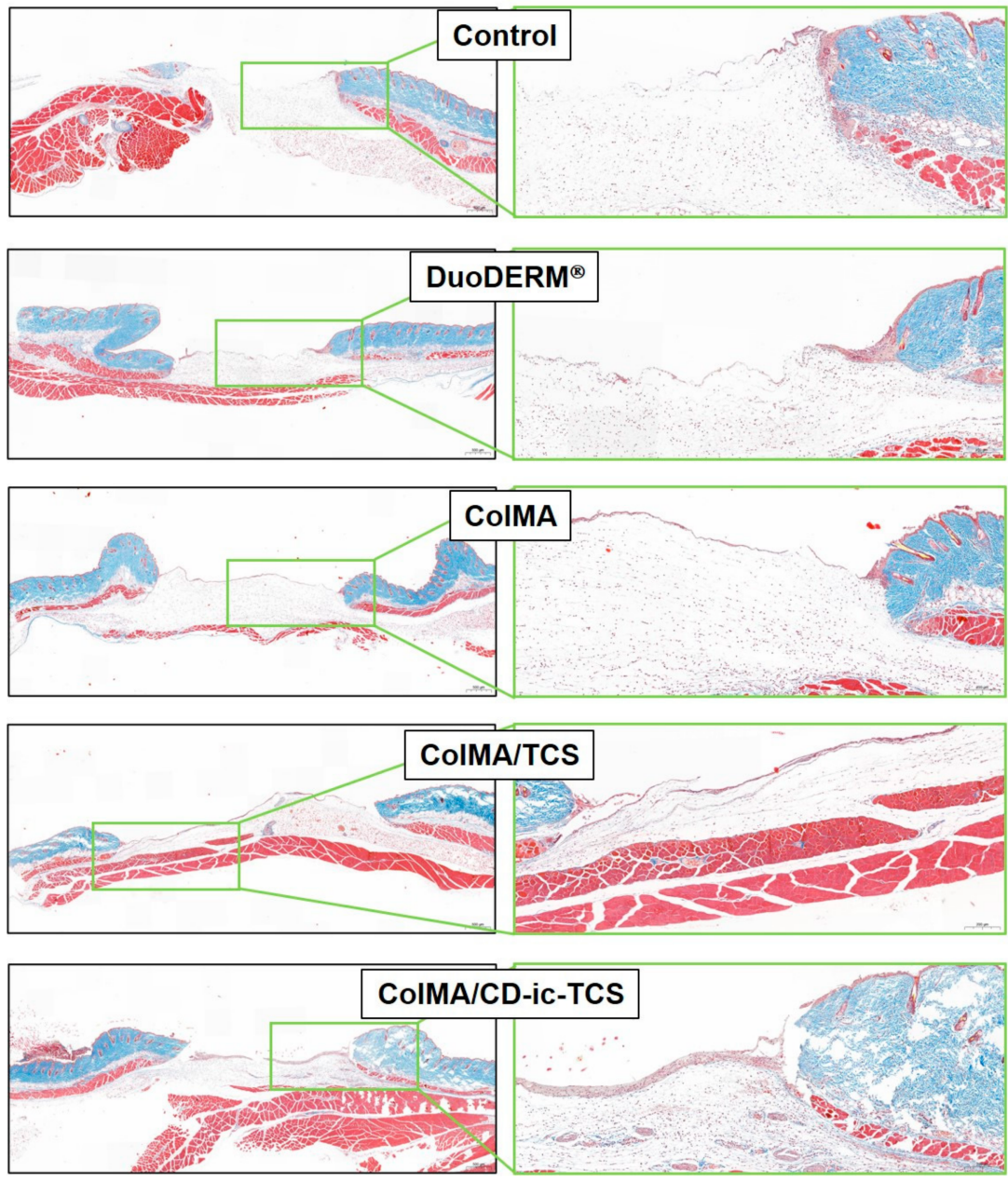

Figure 10. Cont. 

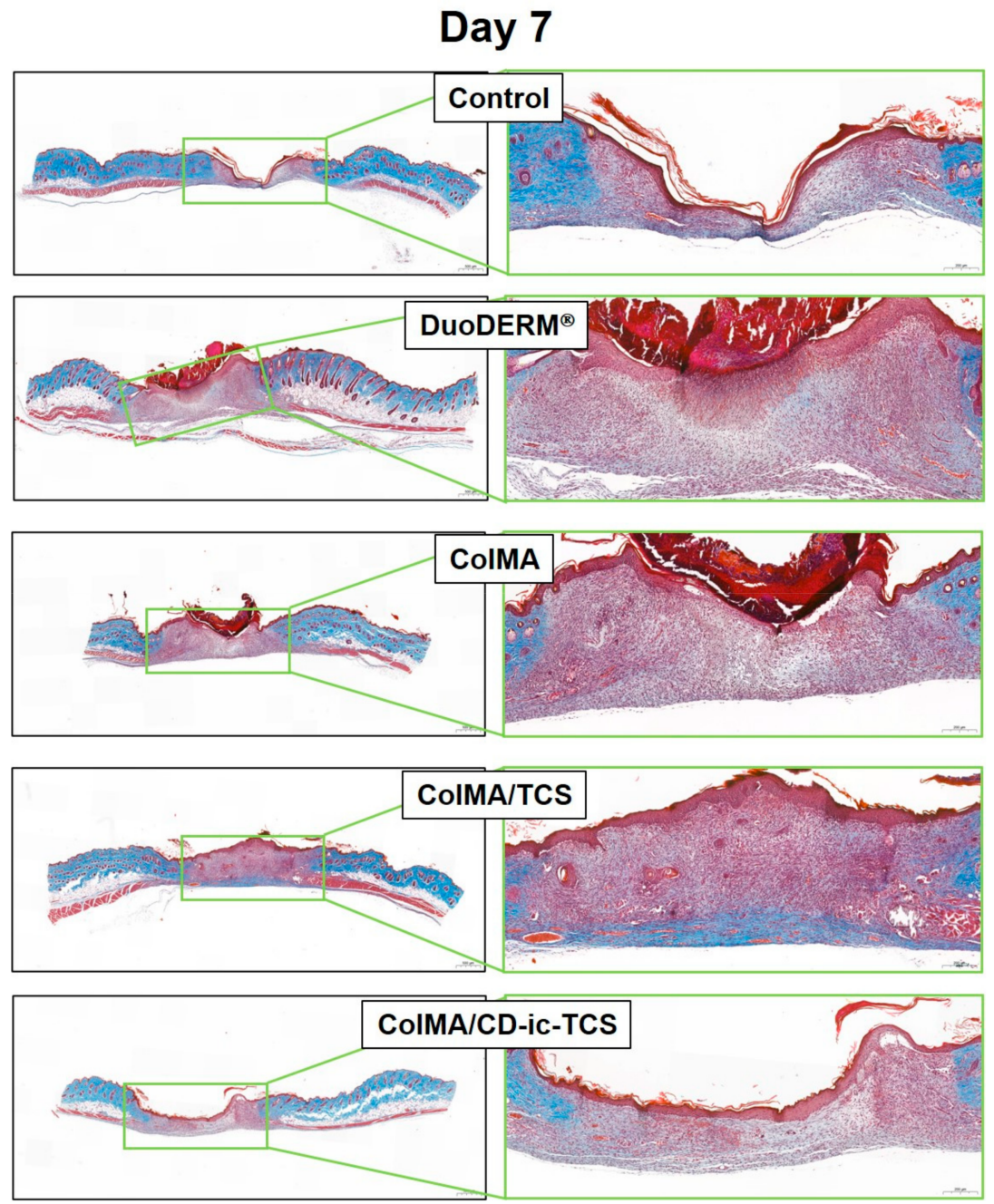

Figure 10. Cont. 


\section{Day 14}
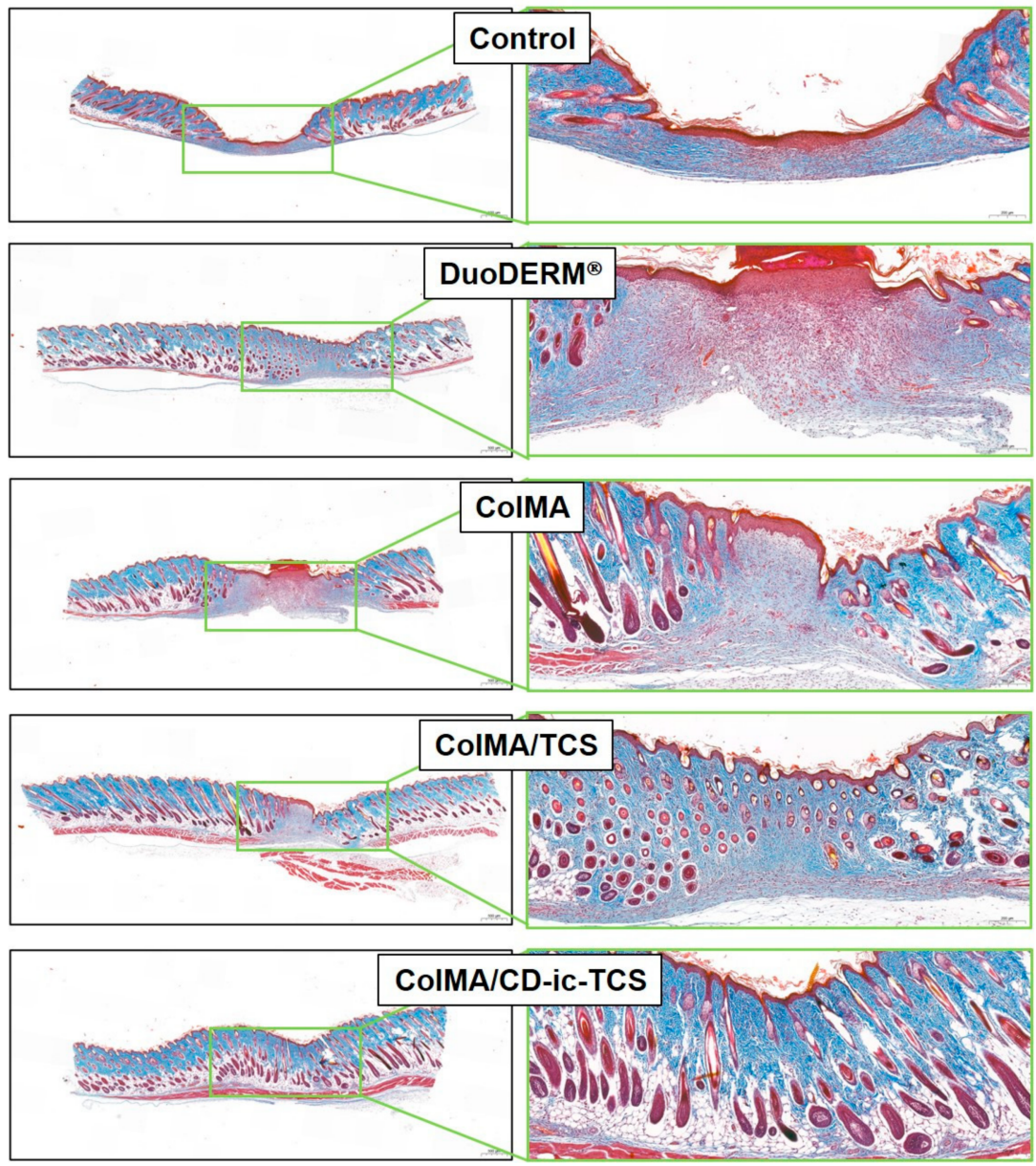

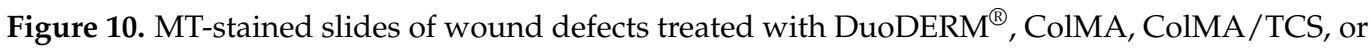
ColMA/CD-ic-TCS for 1, 7, and 14 days. The left and right images were observed at $2.0 \times(500 \mu \mathrm{m})$ and $7.0 \times(200 \mu \mathrm{m})$, respectively. ColMA/TCS—triclosan (TCS) incorporated ColMA; ColMA/CD-icTCS-HP- $\beta-C D / T C S$ complex incorporated ColMA. Blue color indicates collagen.

\section{Discussion}

The ultimate goal of wound healing is a complete healing of the defect without any lasting effects, such as scar formation. One of the causes of scar formation is bacterial invasion. Natural collagen is widely used in research and clinical practice due to its biocompatibility and low antigenicity. However, natural collagen itself is not appropriate as a wound dressing because it can easily be enzymatically degraded. Several types of collagenbased wound dressings, including hydrogels, sheets, and sponges, have been introduced because the physicochemical properties of the polymer allow for facile remodeling [29]. These collagen-based dressings can advance wound healing by inactivating excessive matrix metalloproteases. In addition, collagen promotes migration of healing-related cells towards the wound, which stimulates angiogenesis, re-epithelialization, and new tissue formation [30].

Among dressing types, hydrogel systems can absorb wound exudates because of their 3-D network. In addition, a hydrogel can be used in wounds of various sizes or shapes because of its ability to swell $[3,21,31,32]$. Due to its water-insoluble properties, only a few collagen-based hydrogels for wound dressings have been reported [30,33,34]. However, the collagen hydrogels had uncontrollable mechanical properties; on the other hand, the mechanical properties of the photo-cured collagen hydrogel described in this study may 
be easily controlled by the irradiation time, leading to a custom-made controlled drug delivery system.

In the present study, we developed a collagen-based hydrogel containing TCS as an antibacterial wound dressing (Figure 1). The antibacterial activity of TCS varies according to its concentration. At low concentrations, TCS inhibits the synthesis of fatty acids for of bacterial membranes. At high concentrations, TCS disrupts the bacterial membrane, followed by enhanced antibacterial activity. However, TCS has low bioavailability because of its poor water solubility. We used a photo-curing system to incorporate the TCS into the hydrogel and performed methacrylation of the collagen backbone (ColMA) to prepare a collagen hydrogel (Figure 2) [35]. The inclusion of TCS into $\beta$-CD derivatives improved the water solubility of the drug, which was previously confirmed by UV-vis spectroscopy (Figure 3) [36]. Because TCS can be cytotoxic at concentrations greater than $5 \mu \mathrm{g} / \mathrm{mL}$, we performed in vitro cell-proliferation and antibacterial-activity assays. Our results (Figures 6 and 7) indicated that the ColMA hydrogel was a good material for decreasing the cytotoxicity of TCS and increasing its antibacterial activity [22].

We performed in vivo animal testing to evaluate the feasibility of using ColMA/CD-icTCS to accelerate wound healing. The porosity of the collagen-based hydrogel contributed to the controlled release of TCS. Application of $\beta$-CD to TCS resulted in inclusion complex formation and faster release of TCS. Both ColMA and CD-ic-TCS improved wound healing, according to our assessment of the gross appearance of wounds and the wound sizes (Figures 6-8). Histological evaluations further demonstrated the beneficial effects of the ColMA hydrogel and CD-ic-TCS on wound healing (Figures 9 and 10). After a healing period of 14 days, the mice treated with ColMA/CD-ic-TCS showed complete wound healing. These results suggested that ColMA/CD-ic-TCS promoted wound healing.

\section{Conclusions}

We prepared an antibacterial wound dressing based on a visible light-cured ColMA hydrogel and an HP- $\beta-C D / T C S$ complex and evaluated its wound healing capacity. The photo-cured ColMA hydrogel and HP- $\beta$-CD/TCS were fabricated by photo-curing-using riboflavin as a photoinitiator-and inclusion complex formation, respectively. ColMA/CDic-TCS was characterized by ${ }^{1} \mathrm{H}$ NMR analysis and UV-vis spectroscopy, which confirmed the successful formation of methacrylated collagen and HP- $\beta-C D / T C S$ complex, respectively. In addition, in vitro tests, including SEM, a release test, biocompatibility test, and antibacterial assay, showed that the porous ColMA/CD-ic-TCS exhibited a controlled release of TCS, good biocompatibility, and high antibacterial activity. ColMA/CD-ic-TCS also accelerated wound healing in an in vivo mouse model. Together, our findings suggest that ColMA/CD-ic-TCS has the potential for clinical use as an antibacterial wound dressing.

Author Contributions: Conceptualization, L.J., K.P. and D.H.Y.; methodology, L.J., K.P. and D.H.Y.; formal analysis, Y.Y., H.S.K., H.J.K., J.W.C. and D.Y.L.; data curation, L.J., K.P. and D.H.Y.; writingoriginal draft preparation, L.J., K.P. and D.H.Y.; supervision, D.H.Y. and H.J.C.; funding acquisition, D.H.Y. All authors have read and agreed to the published version of the manuscript.

Funding: This research was supported by the Ministry of Trade, Industry \& Energy (MOTIE, Korea) (20003560 and 20004627; D.H.Y.), the Technology development program (S2782460; D.H.Y.) funded by the Ministry of SMEs and Startups (MSS; Korea).

Institutional Review Board Statement: This study was approved by the Institutional Animal Care and Use Committee of Chung-Ang University (13 April 2020; 2020-00037).

Informed Consent Statement: Not applicable.

Data Availability Statement: Not applicable.

Conflicts of Interest: The authors declare no conflict of interest. 


\section{References}

1. Rezvani Ghomi, E.; Khalili, S.; Nouri Khorasani, S.; Esmaeely Neisiany, R.; Ramakrishna, S. Wound dressings: Current advances and future directions. J. Appl. Polym. Sci. 2019, 136, 47738. [CrossRef]

2. Cutting, K.F. Wound dressings: 21st century performance requirements. J. Wound Care 2010, 19, 4-9. [CrossRef]

3. Yang, D.H.; Seo, D.I.; Lee, D.-W.; Bhang, S.H.; Park, K.; Jang, G.; Kim, C.H.; Chun, H.J. Preparation and evaluation of visible-light cured glycol chitosan hydrogel dressing containing dual growth factors for accelerated wound healing. J. Eng. Ind. Chem. 2017, 53, 360-370. [CrossRef]

4. Taylor, D.L.; in het Panhuis, M. Self-Healing Hydrogels. Adv. Mater. 2016, 28, 9060-9093. [CrossRef]

5. Li, J.; Wu, C.; Chu, P.K.; Gelinsky, M. 3D printing of hydrogels: Rational design strategies and emerging biomedical applications. Mater. Sci. Eng. R Rep. 2020, 140, 100543. [CrossRef]

6. Basha, S.I.; Ghosh, S.; Vinothkumar, K.; Ramehs, B.; Hema Praksh Kumari, P.; Murali Mohan, K.V.; Sukumar, E. Fumaric acid incorporated Ag/agar-agar hybrid hyudrogel: A multifunctional avenue to tackle wound healing. Mater. Sci. Eng. C Mater. Biol. Appl. 2020, 111, 110743. [CrossRef]

7. Mele, A.; Castiglione, F.; Malpezzi, L.; Ganazzoli, F.; Raffaini, G.; Trotta, F.; Rossi, B.; Fontana, A.; Giunchi, G. HR MAS NMR, powder XRD and Raman spectroscopy study of inclusion phenomena in bCD nanosponges. J. Incl. Phenom. Macrocycl. Chem. 2011, 69, 403-409. [CrossRef]

8. Crupi, V.; Majolino, D.; Mele, A.; Rossi, B.; Trotta, F.; Venuti, V. Modelling the interplay between covalent and physical interactions in cyclodextrin-based hydrogel: Effect of water confinement. Soft Matter 2013, 9, 6457. [CrossRef]

9. Singh, G.; Lohani, A.; Bhattacharya, S.S. Hydrogel as a novel drug delivery system: A review. J. Fundam. Pharm. Res. 2014, 2, 35-48.

10. Brandl, F.; Sommer, F.; Goepferich, A. Rational design of hydrogels for tissue engineering: Impact of physical factors on cell behavior. Biomaterials 2007, 28, 134-146. [CrossRef]

11. Doillon, C.J.; Sliver, F.H. Collagen-based wound dressing: Effects of hyaluronic acid and firponectin on wound healing. Biomaterials 1986, 7, 3-8. [CrossRef]

12. Wiegand, C.; Buhren, B.A.; Bünemann, E.; Schrumpf, H.; Homey, B.; Frykberge, R.G.; Lurie, F.; Gerber, P.A. A novel native collagen dressing with advantageous properties to promote physiological wound healing. J. Wound Care 2016, 25, 713-720. [CrossRef]

13. Bendtsen, S.T.; Wei, M. Synthesis and characterization of a novel injectable alginate-collagen-hydroxyapatite hydrogel for bone tissue regeneration. J. Mater. Chem. B 2015, 3, 3081-3090. [CrossRef] [PubMed]

14. Song, K.; Xu, H.; Mu, B.; Xie, K.; Yang, Y. Non-toxic and clean crosslinking system for protein materials: Effect of extenders on crosslinking performance. J. Clean. Prod. 2017, 150, 214-223. [CrossRef]

15. Choi, J.R.; Young, K.W.; Choi, J.Y.; Cowie, A.C. Recent advances in photo-crosslinkable hydrogels for biomedical applications. Biotechniques 2019, 66, 40-53. [CrossRef]

16. Hyun, H.; Park, M.H.J.G.; Kim, S.Y.; Chun, H.J.; Yang, D.H. Photo-cured glycol chitosan hydrogel for ovarian cancer drug delivery. Mar. Drug 2019, 17, 41-52. [CrossRef]

17. Yoon, S.J.; Moon, Y.J.; Chun, H.J.; Yang, D.H. Doxorubicin·hydrochloride/cisplatin-loaded hydrogel/nanosized (2hydroxypropyl)-beta-cyclodextrin local drug-delivery system for osteosarcoma treatment in vivo. Nanomaterials 2019, 9 , 1652-1663. [CrossRef]

18. Yoo, Y.; Yoon, S.J.; Kim, S.Y.; Lee, D.W.; Um, S.; Hyun, H.; Hong, S.O.; Yang, D.H. A local drug delivery system based on visible light-cured glycol chitosan and doxorubicin.hydrochloride for thyroid cancer treatment in vitro and in vivo. Drug Deliv. 2018, 25, 1664-1671. [CrossRef]

19. Hyun, H.; Park, M.H.; Lim, W.; Kim, S.Y.; Jo, D.; Jung, J.S.; Jo, G.; Um, S.; Lee, D.W.; Yang, D.H. Injectable visible light-cured glycol chitosan hydrogels with controlled release of anticancer drugs for local cancer therapy in vivo: A feasible study. Artif. Cells Nanomed. Biotech. 2018, 46, S874-S882. [CrossRef]

20. O'Brien, F.J. Biomaterials \& scaffolds for tissue engineering. Mat. Today 2011, 14, 88-95.

21. Yoo, Y.; Hyun, H.; Yoon, S.-J.; Kim, S.Y.; Lee, D.-W.; Um, S.; Hong, S.O.; Yang, D.H. Visible light-cured glycol chitosan hydrogel dressing containing endothelial growth factor and basic fibroblast growth factor accelerates wound healing in vivo. J. Ind. Eng. Chem. 2018, 67, 365-372. [CrossRef]

22. Jones, R.D.; Jampani, H.B.; Newman, J.L.; Lee, A.S. Triclosan: A review of effectiveness and safety in health care settings. Am. J. Infect. Control 2000, 28, 184-196. [CrossRef] [PubMed]

23. Brinkman, W.T.; Nagapudi, K.; Thomas, B.S.; Chaikof, E.L. Photo-cross-linking of type I collagen gels in the presence of smooth muscle cells: Mechanical properties, cell viability, and function. Biomacromolecules 2003, 3, 890-895. [CrossRef] [PubMed]

24. Fagui, A.E.; Dubot, P.; Loftsson, T.; Amiel, C. Triclosan-loaded with high encapsulation efficiency into PLA nanoparticles coatged with $\beta$-cyclodextrin polymer. J. Incl. Phenom. Macro. Chem. 2013, 75, 277-283. [CrossRef]

25. Moon, Y.J.; Yoon, S.J.; Koo, J.H.; Yoo, Y.; Byun, H.J.; Kim, H.S.; Khang, G.; Chun, H.J.; Yang, D.H. $\beta$-Cyclodextrin/triclosan complex-grafted methacrylated glycol chitosan hydrogel by photocrosslinking via visible light irradiation for a tissue bio-adhesive. Int. J. Mol. Sci. 2021, 22, 700. [CrossRef]

26. Chen, H.; Cheng, J.; Ran, L.; Yu, K.; Lu, B.; Lan, G.; Dai, F.; Lu, F. An injectable self-healing hydrogel with adhesive and antibacterial properties effectively promotes wound healing. Carbohydr. Polym. 2018, 201, 522-531. [CrossRef] 
27. Maniyazagan, M.; Chakraborty, S.; Pérez-Sánchez, H.; Stalin, T. Encapsulation of triclosan within 2-hydroxypropyl- $\beta$-cyclodextrin cavity and its application in the chemisorption of rhodamine B dye. J. Mol. Liq. 2019, 282, 235-243. [CrossRef]

28. Kacsó, I.; Borodi, G.; Farcas, S.I.; Hernanz, A.; Bratu, I. Host-guest system of vitamin B10 in $\beta$-cyclodextrin: Characterization of the interaction in soluytion and in solid state. J. Incl. Phenom. Macrocycl. Chem. 2010, 68, 175-182. [CrossRef]

29. Chattopadhyay, S.; Rains, R.T. Collagen-based biomaterials for wound healing. Biopolymers 2014, 101, 821-833. [CrossRef]

30. Lei, J.; Chen, P.; Li, Y.; Wang, X.; Tang, S. Collagen hydrogel dressing for wound healing and angiogenesis in diabetic rat models. Int. J. Clin. Exp. Med. 2017, 10, 16319-16327.

31. Tavakoli, S.; Klar, A.S. Advanced Hydrogels as Wound Dressings. Biomolecules 2020, 10, 1169. [CrossRef]

32. Yoon, S.-J.; Hyun, H.; Lee, D.-W.; Yang, D.H. Visible light-cured glycol chitosan hydrogel containing a beta-cyclodextrin-curcumin inclusion complex improves wound healing in vivo. Molecules 2017, 22, 1513. [CrossRef]

33. Ying, H.; Zhou, J.; Wang, M.; Su, D.; Ma, Q.; Lv, G.; Chen, J. In situ formed collagen-hyaluronic acid hydrogel as biomimetic dressing for promoting spontaneous wound healing. Mat. Sci. Eng. C 2019, 101, 487-498. [CrossRef]

34. Bai, Z.; Dan, W.; Yu, G.; Wang, Y.; Chen, Y.; Huang, Y.; Yang, C.; Dan, N. Tough and tissue-adhesive polyacrylamide/collagen hydrogel with dopamine-grafted oxidized sodium alginate as crosslinker for cutaneous wound healing. RSC Adv. 2018, 8, 42123-42132. [CrossRef]

35. Nguyen, T.U.; Watkins, K.E.; Kishore, V. Photochemically crosslinked cell-laden methacrylated collagen hydrogels with high cell viability and functionality. J. Biomed. Mater. Res. A 2019, 107, 1541-1550. [CrossRef]

36. Peila, R.; Vineis, C.; Varesano, A.; Ferri, A. Different methods for $\beta$-cyclodextrin/triclosan complexation as antibacterial treatment of cellulose substrates. Cellulose 2013, 20, 2115-2123. [CrossRef] 